\section{Prevalence, Treatment, and Outcome of Infection Due to Extended-Spectrum $\beta$-Lactamase-Producing Microorganisms}

TO THE EDITOR-We read with interest the article by Kang et al.' and would like to report the prevalence, treatment, and outcome of nosocomial infections caused by extended-spectrum $\beta$-lactamase (ESBL)-producing Escherichia coli and Klebsiella pneumoniae at a tertiary care center in Thailand. At Thammasart University Hospital (Pratumthani, Thailand), routine laboratory-based detection and confirmation of ESBL-producing E. coli and $K$. pneumoniae began on October 1, 2003. All microbiological detection and confirmation methods were performed according to criteria of the Clinical and Laboratory Standards Institute (formerly, the NCCLS). ${ }^{2}$ To evaluate the baseline prevalence, treatment, and outcomes among hospitalized adults with ESBL-producing $E$. coli or $K$. pneumoniae nosocomial infections, a retrospective study was conducted from October 1, 2003 through July 31, 2004. Centers for Disease Control and Prevention definitions for nosocomial infections were used in the diagnosis of all infections. ${ }^{3}$ The primary outcome was 30-day mortality. Available exposure data included patients' demographic characteristics, comorbidities, severity of illness (based on the Acute Physiology and Chronic Health Evaluation II score), sources of nosocomial infections, empirical antimicrobial regimens received, and the time to initiation of effective antimicrobial therapy (ie, the interval between the first positive culture result and the initiation of carbapenem therapy).

During the 9-month study period, ESBL production was confirmed in $160(26.3 \%)$ of $448 \mathrm{E}$. coli culture isolates and $50(21 \%)$ of $240 \mathrm{~K}$. pneumoniae culture isolates. A total of $100 \%$ of the ESBL-producing clinical isolates were susceptible to imipenem, $60 \%$ to amikacin, and $43 \%$ to ciprofloxacin. Clinically, 110 patients had a nosocomial infection with ESBLproducing E. coli or K. pneumoniae; 90 (81.8\%) had medical records available for further clinical evaluation. Twenty-four (27\%) of these 90 patients developed nosocomial bloodstream infection (BSI) due to ESBL-producing E. coli or K. pneumoniae. Of these patients, $21(87 \%)$ developed secondary nosocomial BSI, including urinary tract infection (in 15 patients), pneumonia (in 5), and surgical site infection (in 1),

TA B LE. Demographic and Clinical Characteristics of 90 Patients With Extended-Spectrum $\beta$-Lactamase-Producing Escherichia coli or Klebsiella pneumonia Nosocomial Bloodstream Infection (BSI) at a Tertiary Care Center in Thailand

\begin{tabular}{|c|c|c|c|c|}
\hline Characteristic & $\begin{array}{l}\text { Total cohort } \\
\qquad(n=90)\end{array}$ & $\begin{array}{c}\text { Survivors } \\
\text { of BSI } \\
(n=12) \\
\end{array}$ & $\begin{array}{c}\text { Nonsurvivors } \\
\text { of BSI } \\
(n=12)\end{array}$ & $P$ \\
\hline Male sex & $34(38)$ & $5(42)$ & $7(58)$ & .68 \\
\hline Age, median years (range) & $63(17-97)$ & $61(30-93)$ & $65(23-89)$ & .85 \\
\hline \multicolumn{5}{|l|}{ Underlying disease } \\
\hline Liver cirrhosis & $7(8)$ & $2(17)$ & $1(8)$ & 1.0 \\
\hline End-stage renal disease & $10(11)$ & $2(17)$ & $2(17)$ & 1.0 \\
\hline Diabetes & $28(31)$ & $3(25)$ & $2(17)$ & 1.0 \\
\hline Any immunocompromised state $\mathrm{e}^{\mathrm{a}}$ & $8(8)$ & $2(17)$ & $2(17)$ & 1.0 \\
\hline APACHE II score, median (range) & $10(2-22)$ & $11(3-15)$ & $12(3-18)$ & .82 \\
\hline \multicolumn{5}{|l|}{ Type of nosocomial infection } \\
\hline Bloodstream infection & $24(26.7)$ & $12(50)$ & $12(50)$ & 1.0 \\
\hline Urinary tract infection only & $49(54)$ & NA & NA & NA \\
\hline Pneumonia only & $14(16)$ & NA & NA & NA \\
\hline Wound infection only & $3(3.3)$ & NA & NA & NA \\
\hline Other types only & $0(0)$ & NA & NA & NA \\
\hline \multicolumn{5}{|l|}{ Empirical antimicrobial therapy received } \\
\hline Carbapenems & $14(17)$ & $4(33)$ & $2(18)$ & .67 \\
\hline Quinolones & $8(9)$ & $2(18)$ & $1(8)$ & 1.0 \\
\hline Third- or fourth-generation cephalosporins & $38(42)$ & $4(33)$ & $5(40)$ & 1.0 \\
\hline$\beta$-Lactam or $\beta$-lactamase inhibitor & $12(13)$ & $1(8)$ & $1(8)$ & 1.0 \\
\hline Aminoglycosides & $6(6)$ & $1(8)$ & $2(18)$ & 1.0 \\
\hline Other & $12(13)$ & $0(0)$ & $1(8)$ & 1.0 \\
\hline Received effective initial antimicrobial therapy & $37(41)$ & $8(67)$ & $5(42)$ & 0.41 \\
\hline 30-day mortality & $26(29)$ & NA & $12(100)$ & NA \\
\hline
\end{tabular}

NOTE. Data are no. (\%) of subjects, unless otherwise indicated. APACHE = Acute Physiology and Chronic Health Evaluation; NA = not applicable; NS = not significant.

a Includes subjects with any type of currently active malignancy, human immunodeficiency virus type 1 infection, or neutropenia and subjects who were currently receiving steroids or immunosuppressive agents. 
whereas 4 patients developed primary BSI due to ESBL-producing E. coli or K. pneumoniae. Demographic and clinical data, including empirical antimicrobial treatment regimens received and outcomes, are summarized in the Table. The overall 30-day mortality rate was $29 \%$ (26 of 90 patients).

Patients with nosocomial BSI due to ESBL-producing $E$. coli or K. pneumoniae were more likely to die than patients without BSI ( $50 \%$ vs $21 \% ; P=.01$ ). There were no significant differences in survival according to baseline demographic or clinical characteristics (Table). Adjustment for severity of illness revealed that mortality was higher among patients who did not receive a carbapenem for at least 2 days during the 5 -day period after the first positive blood culture result than among patients who did (adjusted odds ratio, 2.12; 95\% confidence interval, $1.15-61 ; P=.03$ ). There was no significant difference in mortality among patients who received initial antimicrobial therapy that was effective, compared with patients who received initial antimicrobial therapy that was not effective ( $42 \%$ vs $58 \% ; P=.41$ ), nor was there a significant difference in mortality for patients who received empirical antimicrobial therapy with a carbapenem, compared with patients who received a noncarbapenem regimen (33\% vs 55\%; $P=.67$ ). There was also no difference with respect to patient characteristics, empirical antimicrobial regimens received, or 30-day mortality between patients infected with ESBL-producing $E$. coli and patients infected with ESBL-producing $K$. pneumoniae.

Debate continues on the role of screening clinically relevant gram-negative bacteria for ESBL production, and there are varied interpretations of the impact of this laboratory-based practice on health outcomes and resource use. ${ }^{4}$ Recent studies of treatment failure have called into question the usefulness of screening patients for pathogens with susceptibility to extended-spectrum cephalosporins and the use of carbapenems for treatment of serious infections due to ESBL-producing pathogens. ${ }^{1,5-7}$ Given the high prevalence and associated crude mortality rate associated with ESBL-producing $E$. coli and $K$. pneumoniae BSIs in our study, tertiary care centers in Thailand should consider routine screening of E. coli and K. pneumoniae isolates for ESBL production. Although the small sample size limits our ability to quantify the true risk associated with ineffective antimicrobial therapy or to identify additional factors that may be associated with mortality, our data further substantiate the findings by Kang et al. ${ }^{1}$ and suggest that initial empirical antimicrobial therapy that is ineffective may not be associated with higher mortality rates for nosocomial BSI caused by ESBL-producing E. coli or $K$. pneumoniae. Additional studies on the prevalence, treatment, and role of ineffective antimicrobial therapy on the outcomes for persons infected with ESBL-producing $E$. coli or $K$. pneumoniae in Asian-Pacific countries are needed.

\section{ACKNOWLEDGMENT}

This study was funded by a grant from the Infectious Diseases Association of Thailand (to A.A.).

Anucha Apisarnthanarak, MD; Linda M. Mundy, MD

Dr. Apisarnthanarak is from the Division of Infectious Diseases, Faculty of Medicine, Thammasart University Hospital, Pratumthani, Thailand. Dr. Mundy is from the Saint Louis University School of Public Health, St. Louis, Missouri.

Address reprint requests to Anucha Apisarnthanarak, MD, Division of Infectious Diseases, Faculty of Medicine, Thammasart University Hospital, Pratumthani, Thailand 12120 (anapisarn@yahoo.com).

Infect Control Hosp Epidemiol 2006; 27:326-327

(C) 2006 by The Society for Healthcare Epidemiology of America. All rights reserved. 0899-823X/2006/2703-0023\$15.00.

\section{REFERENCES}

1. Kang CI, Kim SH, Kim DM, et al. Risk factors for and clinical outcomes of bloodstream infection caused by extended-spectrum beta-lactamaseproducing Klebsiella pneumoniae. Infect Control Hosp Epidemiol 2004; 25 : 860-867.

2. NCCLS. Performance Standards for Antimicrobial Susceptibility Testing. 14th Informational supplement. Wayne, PA: NCCLS; 2004. NCCLS document M100-S14.

3. Horan TC, Gaynes RP, Martone WJ, Jarvis WR, Emori TG. CDC definitions for nosocomial infections. Am J Infect Control 1992; 20:271-4.

4. Emery CL, Weymouth LA. Detection and clinical significance of extendedspectrum beta-lactamases in a tertiary-care medical center. J Clin Microbiol 1997; 35:2061-2067.

5. Karas JA, Pillay DG, Muckart D, Sturm AW. Treatment failure due to extended spectrum beta-lactamases. J Antimicrob Chemother 1996; 37:203204.

6. Jorgensen JH, Turnidge JD, Washington JA. Antibacteria susceptibility tests: dilution and disk diffusion methods. In: Murray PR, Baron EJ, Pfaller MA, Tenover FC, Yolken RH, eds. Manual of Clinical Microbiology. 7th ed. Washington, DC: American Society of Microbiology; 1999.

7. Paterson DL, Ko WC, Von Gottberg A, et al. Outcome of cephalosporin treatment for serious infections due to apparently susceptible organisms producing extended-spectrum beta-lactamases: implications for the clinical microbiology laboratory. J Clin Microbiol 2001; 39:2206-2012. 